\title{
Physical Examination Variables Predict Response to Conservative Treatment of Nonchronic Plantar Fasciitis: Secondary Analysis of a Randomized, Placebo-Controlled Footwear Study
}

\author{
James S. Wrobel, DPM, MS, Adam E. Fleischer, DPM, MPH, \\ Jonathon Matzkin-Bridger, DPM, Jeanna Fascione, DPM, Ryan T. Crews, MS, \\ Nicholas Bruning, BS, Beth Jarrett, DPM
}

\begin{abstract}
Background: Plantar fasciitis is a common, disabling condition, and the prognosis of conservative treatment is difficult to predict. Objective: To determine whether initial clinical findings could help predict patient response to conservative treatment that primarily consisted of supportive footwear and stretching.

Setting: Patients were recruited and seen at 2 outpatient podiatric clinics in the Chicago, Illinois, metropolitan area.

Patients: Seventy-seven patients with nonchronic plantar fasciitis were recruited. Patients were excluded if they had a heel injection in the previous 6 months or were currently using custom foot orthoses at the time of screening. Sixty-nine patients completed the final follow-up visit 3 months after receiving the footwear intervention.

Methods: Treatment failure was considered a $<50 \%$ reduction in heel pain at 3 month follow-up. Logistic regression models evaluated the possible association between more than 30 clinical and physical examination findings prospectively assessed at enrollment, and treatment response.

Results: Inability to dorsiflex the ankle past $-5^{\circ}$ (odds ratio [OR] 3.9, $P=.024$ ), nonsevere ( $\leq 7$ on ordinal scale) first-step pain (OR 3.8, $P=.021)$, and heel valgus in relaxed stance (OR 4.0, $P=.014$ ) each predicted treatment failure in multivariable analysis (receiver operating characteristic area under the curve $=.769$ ). Limited ankle dorsiflexion also correlated with greater heel pain severity at initial presentation $(r=-0.312, P=.006)$.

Conclusions: Patients with severe ankle equinus were nearly 4 times more likely to experience a favorable response to treatment centered on home Achilles tendon stretching and supportive therapy. Thus, earlier use of more advanced therapies may be most appropriate in those presenting without severe ankle equinus or without severe first step pain. The findings from our study may not be clinically intuitive because patients with less severe equinus and less severe pain at presentation did worse with conservative care.
\end{abstract}

\section{Introduction}

Plantar fasciitis is the most common cause of plantar heel pain [1,2] and is an ongoing recognized economic burden. It is commonly defined as an overload injury of the proximal plantar fascia at the infracalcaneal insertion. Some studies suggest plantar fasciitis is more common in athletes [3] and those who are obese [4]. Others report it affects approximately $10 \%$ of both the general and running populations [1]. In the United States, it accounts for nearly 1 million patient visits each year [5], and direct costs associated with prescription therapy and outpatient visits alone exceed 284 million dollars annually [6]. Plantar fasciitis has a significant negative impact on general health-related quality of life that is independent of body mass index [7].

The primary etiology of plantar fasciitis is unknown, and there is not a large body of evidence supporting one treatment over another. In the vast majority of cases, symptoms are resolved within a year of onset, regardless of treatment type [8]. There are multiple treatment options, available including stretching, foot orthoses, corticosteroid injections, night splints, extracorporeal shock wave therapy, and surgery [9]; however, it is 
generally accepted that fewer than $10 \%$ of patients will require surgery [10]. Considerable research exists on the effectiveness of various treatments [11-15] and on factors associated with the development of plantar fasciitis [16-19]. Evidence for factors predictive of a positive or negative outcome with the use of supportive foot therapy and patient-directed interventions for plantar fasciitis, however, is absent from the current literature.

With the more recent paradigm shift that is starting in the United States health care system that supports value-based payment dependent on patient outcomes, it is imperative this condition be treated in a more efficient and effective manner. The primary aim of this study was to determine whether clinical findings observed during initial presentation can be predictive of treatment response in patients receiving conservative, supportive therapy for nonchronic plantar fasciitis. A secondary aim was to determine which, if any, baseline observations are associated with degree of functional impairment and/or heel pain severity.

\section{Materials and Methods}

\section{Participants}

This was a secondary data analysis of 77 subjects who participated in a randomized controlled trial (ClinicalTrials.gov Identifier NCT00765843) to evaluate the efficacy of 3 different foot orthoses conditions for the treatment of proximal plantar fasciitis [20]. All participants were ambulatory adult men and women with nonchronic heel pain (ie, symptoms for less than 12 months). All subjects were recruited from 2 podiatry specialty clinics located in the greater Chicago metropolitan area. The data analysis received an exempt status determination from the Institutional Review Board.

All included patients had pain at the plantar fascial attachment to calcaneal tubercle and/or pain distal from the tubercle along the plantar fascial band with typical poststatic dyskinesia or pain with activity after rest. Patient history, physical examination, plain radiograph assessment, and diagnostic ultrasound were used to rule out other etiologies of heel pain, including proximal or local nerve entrapment, arthritis, bone cyst or tumor, or stress fracture.

Patients with a previous heel injection within the past 6 months or who were currently using custom foot orthoses were excluded. Patients with any of the following pathologies also were excluded: proximal musculoskeletal pathology (eg, knee or hip arthritis, sciatica secondary to back pathology, significant limb length discrepancy), use of gait assistive devices (eg, crutches, canes, walkers), inability to wear supportive closed toed shoes, or lack of range motion at the first metatarsophalangeal or subtalar joints.

\section{Overall Study Design}

Details regarding the full randomized controlled trial can be found in a previous publication [20]. To summarize, 77 patients met the eligibility criteria and agreed to participate. Patients were randomized at their screening visit into 1 of 3 treatment groups: custom foot orthoses, prefabricated orthoses, or sham orthoses. Participants and investigators were blinded to group allocation. Blinding was possible by using the same 3-mm neoprene top cover on each type of orthoses and providing each subject with standardized shoes (Brooks Dyad, Brooks Sports Inc., Bothell WA) in which the orthoses were to be worn.

All participants received a standardized baseline assessment, including a comprehensive lower extremity biomechanical examination during the screening/ enrollment visit from a single examiner. The same single examiner also measured the shoes, casted the patient, fit, and dispensed the orthoses and shoes. Treatment also was instituted at that time. Participants were evaluated again at 1 and 3 months for follow-up. Those patients reporting less than $50 \%$ improvement in their heel pain symptoms (using an average of their ordinal pain scale first step and end-of-day pain scores) at 3-month follow-up were classified as having had an unfavorable treatment response, whereas those reporting greater than or equal to $50 \%$ improvement were classified as having had a favorable treatment response.

\section{Assessments}

Each participant completed an ordinal pain scale (1-10) first step pain, end-of-day pain, and Foot Function Index-Revised (FFI-R) surveys regarding their study foot at enrollment. These scales are recognized as reliable and valid outcomes measures [11,21,22]. Patients completed these instruments in a private examination room during the visit. Information regarding other potential covariates including, but not limited to, age, gender, duration of symptoms, height, weight, laterality, foot type, duration of symptoms, and coexisting medical conditions also was recorded (Table 2).

Additionally, a standardized lower extremity biomechanical examination was performed by a single podiatric physician with greater than 20 years of experience (B.J.). The biomechanical examination measurements were performed via the techniques described (B.J.) [23]. The measurements were performed with a standard goniometer, with all measurements being done offweight-bearing except the stance positions (relaxed and neutral calcaneal stance positions and tibial influence). Limb length was measured off-weight-bearing with a standard measuring tape, and the physician measured the anterior superior iliac spine to the medial malleolus and the umbilicus to the medial malleolus.

In addition, visual gait analysis was performed to facilitate identification of asymmetries consistent with 
limb length inequality. Ankle joint dorsiflexion, specifically, was measured off-weight-bearing both with the knee flexed and the knee extended. The patient was placed in a supine position, and the subtalar joint was slightly supinated. A goniometer was aligned such that one arm of the goniometer bisected the shaft of the fibula on the lateral side of the leg, and the other arm was aligned with the lateral border of the foot. The ankle joint was manually dorsiflexed to first resistance. Ankle joint dorsiflexion with the knee extended was measured first.

The physical examination variables assessed are provided in Table 3. In addition, we explored for different foot types previously believed to be important in plantar fasciitis and favorable treatment response. These included hallux limitus [24], functional hallux limitus, hypermobile 1st ray, compensated forefoot varus, and uncompensated rearfoot varus. Participants again completed all questionnaires regarding their study foot at their 1- and 3-month follow-up visits.

\section{Conservative Treatment Protocol}

Outside of the type of foot orthoses and shoes received, all patients were placed on the same patientdirected heel pain treatment protocol [9]. During the screening process participants were casted for orthoses and provided removable longitudinal and metatarsal pads fashioned from a quarter-inch felt for use until their orthoses were distributed at the baseline visit. One investigator (B.J.) measured and dispensed all study shoes. At baseline, only $11 \%$ of patients presented with appropriately fitting shoes when measured from heelto-ball of foot. Sixty-one percent of patients had one size or greater difference when using heel-to-ball of foot measure. Patients were instructed to wear shoes and foot supports (initially longitudinal and metatarsal pads until orthoses were provided) at all times when ambulating. They were also instructed to avoid going barefoot and to avoid the use of ladders. Patients were asked to perform Achilles tendon stretches using both a stair (Figure 1A) and a wall (Figure 1B, runner's stretch) 3 times daily (typically before or after meals) for a total of 1 minute each. Specifically, participants were instructed to perform an initial passive stretch for 30 seconds followed by a deeper stretch for the remaining 30 seconds. This technique was demonstrated by the examiner, and comprehension of the stretching technique was assessed at each study visit.

Patient adherence was assessed at 1 month and at 3 months' follow-up with a simple questionnaire that requested the number of days the patient performed

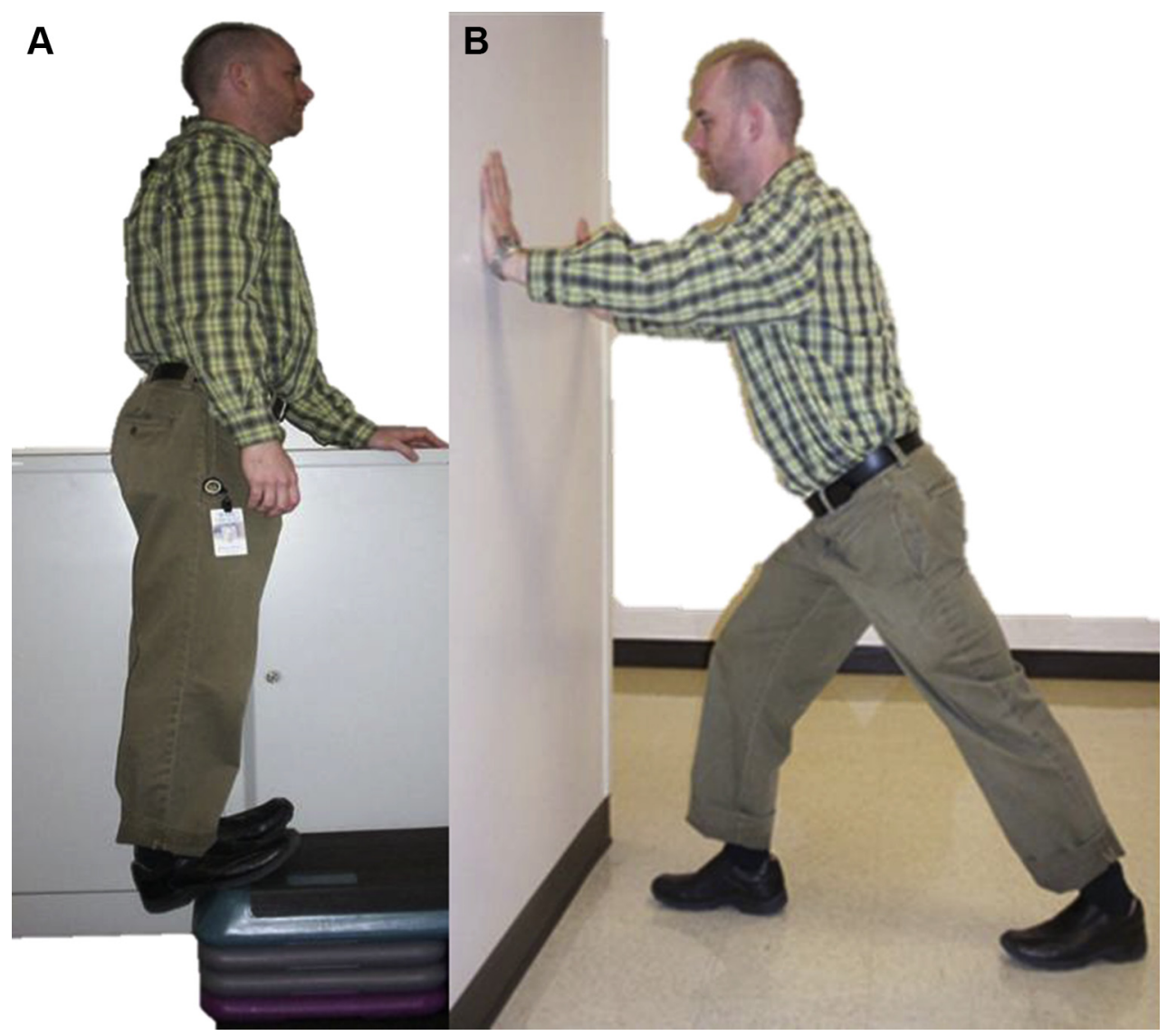

Figure 1. (A) Stair stretch. (B) Runner's stretch. 
stretching and the average number of times per day. Patients also were asked to moderate their recreational activities until their pain had improved. Additionally, they were instructed to avoid nonsteroidal antiinflammatory drugs and instead asked to perform ice massage to the symptomatic heel for 20 minutes per hour, for as long as necessary at the end of the day, as a method of controlling inflammation. Patients also were asked about other anti-inflammatory medication they may have been taking. Written instructions and paper cups for ice massage were provided. A rescue analgesia protocol consisting of supplied acetaminophen was incorporated into the study. Patients were asked to use the acetaminophen as little as possible and were provided a diary to track their use of it. Lastly, patients were notified that if their pain was still severe after 3 months of orthoses use, they would have the option to receive a steroid injection.

\section{Statistical Analysis}

For patients presenting with bilateral heel pain, the study foot was chosen as the more symptomatic foot or, in the case of equal symptoms, the patient's dominant foot was used. Pearson correlation coefficient $(r)$ was used to examine for correlation between the study's continuous clinical covariates and pain level and disability scores. Patient demographic and baseline characteristics were stratified by treatment response (favorable versus unfavorable). Univariate logistic regression models were used to identify candidate variables associated with treatment response. Variables with $P$ value thresholds $<.10$ were tested in the multivariable analysis. Receiver operating characteristic curves were used to identify cut points for continuous candidate variables and these were also tested for significance in multivariable models. The final multivariable model was determined using stepwise logistic regression with $P<.05$ as the criterion for model entry and $P \geq .05$ for removal. Missing values were imputed with the mean value for the study population during the multivariable analysis.

A sensitivity analysis also was performed to evaluate the influence of participants lost to follow-up $(n=8)$, by including them as nonresponders. Although there were no significant baseline differences between those lost to follow-up and those completing the study, the average baseline AM pain was 5.71 versus 4.78 for those that completed, PM pain 5.71 versus 4.68 for those that completed, and age 44.9 years versus 49.6 years for those that completed. All $P$ values less than .05 were considered significant, and all were 2 tailed. Estimates of risk were given by odds ratios and 95\% confidence intervals. SAS (Statistical Analysis System, SAS Institute Inc., Cary, NC) version 9.2 was used for all data analysis.

\section{Results}

\section{Descriptive Statistics}

Sixty-nine of seventy-seven participants (89.6\%) returned for reassessment at 3 months. Characteristics of the study population with complete follow-up data are provided in Table 1. The study population consisted mostly of white women with mildly obese habitus. The average duration of heel pain at initial presentation was slightly more than 5 months $(5.27 \pm 3.2$ months [range 0.25-12]). Twenty-one participants presented with bilateral heel pain $(21 / 69,30 \%)$. Full compliance with stretching (defined as $>80 \%$ of the days) was reported in greater than $50 \%$ of the study population at both 1 -month and 3-month follow-ups (38/66, 58\% [3 missing] and $38 / 68,56 \%$ [ 1 missing], respectively). More than $80 \%$ of the study population stretched on more than half of the study days (53/66, 80\% at 1 month and $57 / 68,84 \%$ at 3 months). Participants admitted to stretching for a mean $2.6 \pm 1.4$ times per day (range $0-10$ ) at 1 -month follow-up and $2.2 \pm 1.3$ times per day (range 1-10) at 3-month follow-up. No participant admitted to using nonsteroidal anti-inflammatory drugs during the study period.

\section{Clinical Findings Associated With Treatment Response}

Table 2 displays the distribution of select baseline characteristics, including foot support allocation and pain/disability scores, stratified by treatment response. Treatment response was not associated with the type (sham, prefabricated, or custom orthoses) of foot orthoses received $(P>.10)$. Table 3 describes the distribution of baseline biomechanical examination variables stratified by treatment response. Of the variables examined in the univariate analysis, hip internal rotation (with hip extended, $P=.008$ ), hip neutral position (with hip extended, $P=.016$ ), relaxed calcaneal stance position $(P=.035)$, heel valgus in relaxed stance $(P=.013)$, ankle joint range of motion

Table 1

Demographic and baseline clinical characteristics of participants with 3-month follow-up $(n=69)$

\begin{tabular}{ll}
\hline Characteristic & Mean \pm SD (Range) \\
\hline Gender & \\
$\quad$ Male & 25 \\
Female & 44 \\
Age, y & $49.7 \pm 12.4(23-75)$ \\
BMl, kg/m ${ }^{2}$ & $31.5 \pm 7.29(19.5-60.8)$ \\
Duration of heel pain, mo & $5.27 \pm 3.2(0.25-12)$ \\
Time spent standing, h/d & $2.9 \pm 2.8(0.25-15)$ \\
Pain level & \\
$\quad$ First step & $6.6 \pm 2.2(2-10)$ \\
End of day & $5.7 \pm 2.3(2-10)$ \\
\hline
\end{tabular}

$\mathrm{BMI}=$ body mass index. 
Table 2

Demographics, historical variables, and baseline pain/disability scores for the study population stratified by treatment response $(n=69)$

\begin{tabular}{lccc}
\hline & $\begin{array}{l}\text { Favorable } \\
\text { Response } \\
(\mathrm{n}=39)\end{array}$ & $\begin{array}{l}\text { Unfavorable } \\
\text { Response } \\
(\mathrm{n}=30)\end{array}$ & $P$ Value \\
\hline Demographics & $50.5(10.9)$ & $48.7(14.2)$ & .565 \\
$\quad$ Age & $25(0.64)$ & $19(0.63)$ & .947 \\
Female gender & $31.6(6.2)$ & $31.5(8.5)$ & .960 \\
BMI & $.69 / .13 / .10 / .08$ & $.69 / .03 / .24 / .03$ & .246 \\
Race/ethnicity & $16(.41)$ & $17(.57)$ & .197 \\
Historical variables & $11(.28)$ & $10(.33)$ & .646 \\
Study foot = right & $5.2(3.4)$ & $5.4(2.9)$ & .757 \\
Bilateral symptoms & $3.0(3.3)$ & $2.8(2.0)$ & .759 \\
Heel pain duration, mo & & $13(.43)$ & .122 \\
Standing time/day, $\mathrm{h}$ & $10(.26)$ & $6(.20)$ & .102 \\
Type of foot support & $15(.38)$ & $11(.37)$ & .947 \\
$\quad$ Sham orthoses & $14(.36)$ & & \\
$\quad$ Prefabricated orthoses & & & \\
$\quad$ Custom orthoses & $7.0(2.2)$ & $6.1(2.2)$ & .095 \\
Pain/Disability Score & $5.8(2.3)$ & $5.7(2.4)$ & .767 \\
Pain first step & $37.1(9.2)$ & $35.8(10.2)$ & .573 \\
Pain end-of-day & $165(45.5)$ & $164(40.5)$ & .919 \\
FFI-R pain subscale & & \\
FFI-R total & &
\end{tabular}

All values are displayed as frequency (percentage of column total) or as mean (SD); Race/ethnicity presented as percentage of column total for Caucasian/African American/Hispanic/Other.

$t$-test or Wilcoxon rank-sum test was used for comparisons of continuous variables.

$\chi^{2}$ or Fisher exact test was used for comparisons of categorical variables.

$\mathrm{BMI}=$ body mass index; FFI-R $=$ Foot Function Index-Revised.

* Logistic regression $P$ value $>.10$.

$<$ negative $5^{\circ}$ (with knee extended, $P=.022$ ), and first step heel pain $\leq 7(P=.031)$ were each associated with treatment response. Other potentially important variables $(P<.10)$ that were tested in the multivariable analysis included baseline first step pain, hip neutral position (with hip flexed), and amount of passive subtalar joint pronation. The only significant predictors of an unfavorable treatment response in the multivariable analysis were having heel valgus in relaxed stance, inability to dorsiflex past $-5^{\circ}$ (with knee extended), and having first step heel pain $\leq 7$ at initial presentation (Table 4$)$. Seventy percent $(22 / 31)$ of participants with severe first step heel pain $(\geq 7 / 10)$ responded to treatment in our study compared to only $45 \%(17 / 38)$ of those without severe heel pain. Seventy-one percent $(25 / 35)$ of participants without heel valgus responded to treatment compared with $41 \%(14 / 34)$ of participants with heel valgus. Sixty-seven percent $(30 / 45)$ of participants with severe ankle equinus $\left(\leq\right.$ neg. $\left.5^{\circ}\right)$ responded compared with only $38 \%(9 / 24)$ of participants with without severe equinus. We found no differences in foot types, including foot posture index, hallux limitus, functional hallux limitus, hypermobile 1st ray, compensated forefoot varus, and uncompensated rearfoot varus (Table 5).
Table 3

Baseline lower extremity biomechanical measurements for the study population stratified by treatment response $(n=69)$

\begin{tabular}{|c|c|c|c|}
\hline & $\begin{array}{l}\text { Favorable } \\
\text { Response } \\
(\mathrm{n}=39)\end{array}$ & $\begin{array}{l}\text { Unfavorable } \\
\text { Response } \\
(\mathrm{n}=30)\end{array}$ & $P$ Value \\
\hline \multicolumn{4}{|l|}{ Hip and gait measurements } \\
\hline Angle of gait & $14.6(5.3)$ & $12.9(5.5)$ & .189 \\
\hline Base of gait, $\mathrm{cm}$ & $7.3(4.4)$ & $6.5(3.4)$ & .416 \\
\hline Limb length diff., $\mathrm{cm}-$ ASIS & $1.5(13.1)$ & $0.0(1.8)$ & .532 \\
\hline Limb length diff., $\mathrm{cm}-$ umb. & $2.1(14.2)$ & $.3(1.7)$ & .503 \\
\hline Hip internal rotation, hip ext. & $16.6(4.9)$ & $19.8(4.8)$ & $.008^{*}$ \\
\hline Hip internal rotation, hip flex & $22.5(5.3)$ & $24.5(4.9)$ & .129 \\
\hline Hip external rotation, hip ext. & $18.6(5.6)$ & $18.1(4.1)$ & .661 \\
\hline Hip external rotation, hip flex & $20.4(5.0)$ & $19.4(4.2)$ & .419 \\
\hline $\begin{array}{l}\text { Hip neutral position, hip ext. } \\
\text { support }\end{array}$ & $-1.0(3.2)$ & $.87(3.1)$ & $.016^{*}$ \\
\hline Hip neutral position, hip flex & $1.1(3.5)$ & $2.5(3.0)$ & .078 \\
\hline Hip abduction & $32.6(3.9)$ & $32.3(3.6)$ & .725 \\
\hline Hip adduction & $21.9(3.6)$ & $21.8(4.5)$ & .942 \\
\hline Hip flexion, knee ext. & $42.1(7.9)$ & $39.7(8.7)$ & .245 \\
\hline Hip flexion, knee flex & $80.0(12.4)$ & $81.4(11.5)$ & .635 \\
\hline Hip extension, knee ext. & $17.7(2.9)$ & $17.8(4.6)$ & .909 \\
\hline Hip extension, knee flex & $18.9(3.4)$ & $18.5(5.2)$ & .727 \\
\hline \multicolumn{4}{|l|}{ Ankle and subtalar joint } \\
\hline Malleolar position & $13.7(2.7)$ & $13.9(3.0)$ & .735 \\
\hline Tibial influence & $1.1(3.0)$ & $.9(3.1)$ & .802 \\
\hline Ankle dorsiflexion, knee flex & $2.6(5.2)$ & $4.1(5.2)$ & .242 \\
\hline Ankle dorsiflexion, knee ext. & $-7.6(4.9)$ & $-5.7(5.1)$ & .121 \\
\hline Subtalar joint supination & $14.5(4.9)$ & $14.3(4.1)$ & .885 \\
\hline Subtalar joint pronation & $3.1(2.7)$ & $4.3(2.3)$ & .069 \\
\hline Subtalar joint neutral pos. & $2.8(2.0)$ & $2.5(2.0)$ & .445 \\
\hline Subtalar joint neutral pos. calc. & $2.7(2.5)$ & $1.9(1.7)$ & .133 \\
\hline Relaxed calcaneal stance pos. ${ }^{\dagger}$ & $-0.7(3.2)$ & $-2.2(2.3)$ & $.035^{*}$ \\
\hline Neutral calcaneal stance pos. & $5.2(2.5)$ & $4.3(3.0)$ & .193 \\
\hline Foot posture index (score) & $4.5(3.5)$ & $5.2(3.0)$ & .391 \\
\hline
\end{tabular}

All values are displayed as mean (SD); all values are reported in degrees unless otherwise specified; all measurements refer to the ipsilateral extremity as the study foot except "base of gait" and "limb length difference."

$t$-test or Wilcoxon rank-sum test was used for comparisons of continuous variables.

ASIS = anterior superior iliac spine; umb. = umbilicus; ext. = extended; flex $=$ flexed; pos. = position; calc. $=$ calculated.

* Statistically significant test result.

† A negative number indicates valgus heel position.

\section{Baseline Observations Associated With Functional Impairment/Heel Pain Severity}

Ankle joint dorsiflexion (with the knee flexed) demonstrated a moderate correlation with pain and a modest correlation with disability at initial presentation. Patients with less ankle joint dorsiflexion generally reported greater end-of-day $(r=-.357, P=.002)$ and greater average heel pain levels $(r=-.312, P=.006)$. Patients with less ankle joint dorsiflexion also tended to report greater total scores (eg, more disability) on FFI-R at presentation $(r=.303, P=.008)$.

BMI demonstrated a modest correlation with both pain and disability scores at initial presentation. Patients with greater BMI tended to report greater levels 
Table 4

Final model for predicting an unfavorable response to conservative therapy for nonchronic plantar fasciitis $(n=69)$

\begin{tabular}{lllll}
\hline Risk Factor* & $\begin{array}{l}\text { Regression } \\
\text { Coefficient }\end{array}$ & $\begin{array}{l}\text { Odds } \\
\text { Ratio }\end{array}$ & $\begin{array}{l}95 \% \text { Confidence } \\
\text { Interval }\end{array}$ & $P$ Value \\
\hline $\begin{array}{l}\text { Intercept } \\
\begin{array}{l}\text { Ankle dorsiflexion } \\
\quad \text { neg. 5 }\end{array}\end{array}$ & $\begin{array}{c}-2.240 \\
\quad 1.368\end{array}$ & - & - & .001 \\
$\begin{array}{c}\text { Heel valgus in } \\
\text { relaxed stance }\end{array}$ & 1.395 & 4.0 & $1.2-12.5$ & .021 \\
\begin{tabular}{l} 
AM pain score $\leq 7$ \\
\hline
\end{tabular} & 1.346 & 3.8 & $1.2-12.2$ & .014 \\
\hline
\end{tabular}

Ordinal pain score (1-10).

The area under the receiver operating characteristic curve for this model $=.769$. Hosmer-Lemeshow goodness of fit test $=1.836$, $P=.934$.

* Observations at baseline (patient's initial presentation); biomechanical observations refer to the ipsilateral extremity as the study foot.

of end-of-day pain $(r=.244, P=.033)$, greater average heel pain levels $(r=.224, P=.049)$, greater scores on the FFI-R pain subscale $(r=.268, P=.019)$, and greater total disability/dysfunction scores on the FFI-R $(r=.303, P=.008)$. Participants with greater pain levels on FFI-R pain subscale also tended to exhibit a narrower base of gait $(r=-.302, P=.008)$.

Finally, Hispanic patients $(n=11)$ reported greater first-step heel pain (mean $8.1 \pm 1.9$ versus $6.5 \pm 2.2$, $P=.026$ ) and greater average heel pain (mean $8.0 \pm 1.5$ versus $6.0 \pm 1.9, P=.002$ ) levels compared with nonHispanic patients. There were no other correlations observed between demographic, historical, and/or physical examination characteristics and magnitude of pain/disability at initial presentation. Furthermore, we explored treatment response thresholds. There were only 9 subjects (of 69) who had no pain (visual analog scale $A M+$ visual analog scale $P M$ pain $=0$ ) at 3 months, and 18 (of 69) that had $80 \%$ improvement or greater at 3 months. Not surprisingly when these outcomes were modeled in the multivariate analysis, there were no significant predictors identified.

In our sensitivity analysis, there were no significant differences in age, baseline AM pain, and baseline PM pain for the lost to follow-up group $(n=8)$ and those who completed the study. When we included the lost to

Table 5

Foot type assessment and foot-specific measures

\begin{tabular}{llll}
\hline Foot Type Measurements & $\begin{array}{l}\text { Favorable } \\
\text { Response } \\
(\mathrm{n}=39)\end{array}$ & $\begin{array}{l}\text { Unfavorable } \\
\text { Response } \\
(\mathrm{n}=30)\end{array}$ & $P$ Value \\
\hline Hallux dorsiflexion unloaded & $44.1(8.7)$ & $44.4(11.1)$ & .898 \\
Hallux dorsiflexion loaded & $32.6(8.5)$ & $32.9(9.8)$ & .746 \\
Functional hallux limitus, diff. & $11.5(6.5)$ & $11.6(6.5)$ & .925 \\
First ray dorsiflexion, mm & $3.3(1.6)$ & $3.5(1.5)$ & .867 \\
First ray plantarflexion, mm & $5.1(2.1)$ & $5.3(1.8)$ & .798 \\
Forefoot varus $(25 \%)$ compensation & $-5.7(4.7)$ & $-7.1(6.1)$ & .438 \\
Rearfoot varus $\left(>4^{\circ}\right)$ & $30 \%$ & $14 \%$ & .164 \\
\hline
\end{tabular}

follow-up group as nonresponders and repeated our logistic model, the effect sizes did not change substantially (data not shown).

\section{Discussion}

Studying a compliant patient population with nonchronic plantar fasciitis, we found that patients with severe ankle joint equinus were more likely to respond favorably to a treatment program that centered on conservative care, including Achilles tendon stretching. Specifically, patients unable to dorsiflex to at least $-5^{\circ}$ (with the knee extended) at initial presentation were nearly 4 times more likely to experience an unfavorable response at 3 months' follow-up. Although equinus is certainly a recognized risk factor for development of plantar fasciitis [25-27], we believe our study is the first to actually conclude that more ankle equinus at the start of therapy translates into improved outcome after conservative therapy [9].

Our findings are encouraging as they suggest, like previous work, many people with nonchronic plantar fasciitis will benefit from conservative care [2]. After all, $65 \%(45 / 69)$ of the participants in our study exhibited a severe ankle joint equinus at their initial presentation, and it is well recognized that many individuals suffering with plantar fasciitis will demonstrate restricted ankle joint motion [16,25-27]. Despite there being a lack of clear evidence surrounding the benefit of Achilles tendon stretching [28], authorities still maintain that home Achilles tendon stretching is an integral part in the conservative management of plantar fasciitis $[29,30]$. Our work may help explain the mixed results of past work. Our protocol relied on stretching, ice massage, standardized shoes, and altering footwear behaviors. Our work also found that stretching alone was not enough for experiencing relief. More specifically, we found that the amount of stretching (ie, days or times per day) determined by patient self-report was not associated with a favorable treatment response $(P>.05)$. Therefore, it may be that it is the combined effect of regular stretching along with an initial severe posterior muscle group tightness that favors a good response to treatment. Our study was underpowered to fully examine this interaction effect, but future work is probably warranted to further explore this effect.

Subjects with a valgus rearfoot position in relaxed stance were also more likely to experience an unfavorable response to treatment. There is some limited evidence that suggests that patients with pronated feet may be more likely to develop symptoms of chronic plantar fasciitis. In a recent case-control study, Irving et al [4] found that patients with chronic heel pain (defined as $>6$ months of symptoms) were nearly 4 times more likely (odds ratio 3.7, 95\% confidence interval 1.6-8.7) to have a pronated foot posture, determined with FPI. Our findings are in close agreement with these results, because 
our patients with nonchronic plantar fasciitis and heel valgus were 4 times less likely to respond to patientdirected treatment measures. These findings make some intuitive sense, because excessive pronation of the foot increases tensile loads on the plantar fascia [31,32]. It is also possible that an everted heel may allow for more displacement of the plantar calcaneal fat pad, and therefore possibly less shock absorption during impact (ie, heel strike). Indeed, we examined our data for other foot types that were poor shock attenuators. We found uncompensated rearfoot varus was prevalent at $30 \%$; however, its association with treatment response only trended towards significance $(P=.12)$.

Outside of heel valgus and a tight gastro-soleal complex, we did not find any other biomechanical measurements associated with treatment response. Although hamstring tightness has been implicated in the development of plantar fasciitis in previous work [33], hip flexion did not seem to correlate with likelihood of achieving a favorable response in our work. This finding was not entirely unexpected; however, because there was no effort made during any of our offered therapies to address a possible contracture of the proximal limb posterior muscles. Further work to address whether therapies aimed at relieving hamstring tightness for plantar fasciitis may be warranted. We were surprised by our findings that hallux limitus or functional hallux limitus were not associated with treatment response, especially given our novel findings of poorly fitting shoes at baseline. When measuring from heel-to-ball of foot, only $11 \%$ presented with appropriately fitting shoes with $61 \%$ having $\geq 1$ size difference when the heel-to-ball of foot measure was used. As the plantar medial fascial band is the widest band of tissue, we were surprised hallux limitus was not associated with treatment response. In a case control study, Aranda and Munuera [34] reported significantly more first metatarsal phalangeal joint dorsiflexion in controls than those with plantar fasciitis $\left(71^{\circ}\right.$ versus $\left.49^{\circ}\right)$.

Finally, we found that patients with severe $(>7 / 10)$ first-step heel pain were approximately 4 times more likely to respond favorably to conservative treatment measures. This means that patients presenting for treatment of mild-to-moderate symptoms were less responsive to conservative therapies for heel pain. In a largely self-limiting condition with a high placebo treatment effect [1], it is not surprising to see that patients with the greatest pain may be more likely to experience relief with the introduction of relatively straight-forward program of footwear, insole, and gentle stretching recommendations.

This work also identified several variables that are, at least, modestly associated with greater amounts of pain and/or disability in patients presenting with plantar fasciitis. We found that patients with greater amounts of equinus and greater BMI also were more likely to present with greater average heel pain levels and more self-reported foot disability. These findings add further supportive evidence to the notion that ankle equinus and BMI may be important risk factors in the causal pathway for developing plantar fasciitis $[4,27]$.

The primary strength of this study is that it is the first to look at presenting factors that might be predictive of treatment response in patients suffering with nonchronic plantar fasciitis. Because pain response was not associated with the type of foot orthoses received, we were able to readily evaluate factors that were predictive of pain response independent of treatment allocation. The complete examination performed prospectively at presentation by a single experienced physician allowed for the consideration of many variables. because all subjects received the same shoes and the same instructions on stretching and icing, those aspects were standardized.

We recognize limitations exist and should be addressed in future studies. The primary weakness of the study is that it is a secondary analysis. It would be beneficial to reproduce these findings by the use of a prospective design and in another, larger patient population. Additionally, ankle equinus was only measured during the screening session. It would be interesting to follow potential flexibility improvements in all participants after the initiation of the included stretching protocol. It would also be valuable to look at the potential additive benefit that might be achieved with greater stretching in those that exhibit the greatest posterior group tightness. All of the measures were determined by a sole examiner. Intrarater reliability for measures of the foot and ankle tend to be quite greater than interrater reliability [35]. However, many of these measures do not have published clinical effect sizes to assist in determining the clinical significance of our findings. Lastly, there were a substantially greater number of female participants and the potential effect of this is unknown.

\section{Conclusion}

Patients with severe ankle equinus were nearly 4 times more likely to experience a favorable response to a plantar fasciitis treatment program that centered on a home Achilles tendon stretching regimen and conservative therapy. Patients presenting with more pronated foot types and less severe presenting pain were also less likely to respond to conservative care consisting of ankle stretching, ice massage, standardized shoes, and altering footwear behaviors.

\section{References}

1. Riddle DL, Schappert SM. Volume of ambulatory care visits and patterns of care for patients diagnosed with plantar fasciitis: A national study of medical doctors. Foot Ankle Int 2004;25:303-310. 
2. Tisdel CL, Donley BG, Sferra JJ. Diagnosing and treating plantar fasciitis: A conservative approach to plantar heel pain. Cleve Clin J Med 1999;66:231-235.

3. Glazer JL. An approach to the diagnosis and treatment of plantar fasciitis. Phys Sportsmed 2009;37:74-79.

4. Irving DB, Cook JL, Young MA, Menz HB. Obesity and pronated foot type may increase the risk of chronic plantar heel pain: A matched case-control study. BMC Musculoskelet Disord 2007;8:41.

5. Riddle DL, Schappert SM. Volume of ambulatory care visits and patterns of care for patients diagnosed with plantar fasciitis: A national study of medical doctors. Foot Ankle Int 2004;25: 303-310.

6. Tong KB, Furia J. Economic burden of plantar fasciitis treatment in the United States. Am J Orthop (Belle Mead NJ) 2010; $39: 227-231$

7. Irving DB, Cook JL, Young MA, Menz HB. Impact of chronic plantar heel pain on health-related quality of life. J Am Podiatr Med Assoc 2008;98:283-289.

8. Buchbinder R. Clinical practice. Plantar fasciitis. N Engl J Med 2004;350:2159-2166.

9. Thomas JL, Christensen JC, Kravitz SR, et al. The diagnosis and treatment of heel pain: A clinical practice guideline-revision 2010. J Foot Ankle Surg 2010;49(3 Suppl):S1-S19.

10. Neufeld SK, Cerrato R. Plantar fasciitis: Evaluation and treatment. J Am Acad Orthop Surg 2008;16:338-346.

11. Bennett PJ, Patterson C, Wearing S, Baglioni T. Development and validation of a questionnaire designed to measure foot-health status. J Am Podiatr Med Assoc 1998;88:419-428.

12. Collins N, Bisset L, McPoil T, Vicenzino B. Foot orthoses in lower limb overuse conditions: A systematic review and meta-analysis. Foot Ankle Int 2007;28:396-412.

13. Helbig K, Herbert C, Schostok T, Brown M, Thiele R. Correlations between the duration of pain and the success of shock wave therapy. Clin Orthop Relat Res 2001;387:68-71.

14. Martin JE, Hosch JC, Goforth WP, Murff RT, Lynch DM, Odom RD. Mechanical treatment of plantar fasciitis. A prospective study. J Am Podiatr Med Assoc 2001;91:55-62.

15. Schulhofer SD. Short-term benefits of ultrasound-guided corticosteroid injection in plantar fasciitis. Clin J Sport Med 2013;23:83-84.

16. Bolivar YA, Munuera PV, Padillo JP. Relationship between tightness of the posterior muscles of the lower limb and plantar fasciitis. Foot Ankle Int 2013;34:42-48.

17. Fabrikant J, Park T. Plantar fasciitis (fasciosis) treatment outcome study: Plantar fascia thickness measured by ultrasound and correlated with patient self-reported improvement. Foot 2012;21: 79-83.

18. Klein SE, Dale AM, Hayes MH, Johnson JE, McCormick JJ, Racette BA. Clinical presentation and self-reported patterns of pain and function in patients with plantar heel pain. Foot Ankle Int 2012;33:693-698.

19. Labovitz JM, Yu J, Kim C. The role of hamstring tightness in plantar fasciitis. Foot Ankle Spec 2011;4:141-144.

20. Wrobel J, Fleischer AE, Crews RT, Jarrett B, Najafi B. A randomized controlled trial of custom foot orthoses for the treatment of plantar heel pain: A return to spontaneous physical activity. J Am Podiatr Med Assoc 2015;105:281-294.

21. Budiman-Mak E, Conrad K, Stuck R, Matters M. Theoretical model and Rasch analysis to develop a revised Foot Function Index. Foot Ankle Int 2006;27:519-527.

22. Reips UD, Funke F. Interval-level measurement with visual analogue scales in Internet-based research: VAS Generator. Behav Res Methods 2008;40:699-704.

23. Jarrett B. The Biomechanical Examination. In: Albert SF, Curran SA, eds. Lower Extremity Mechanics:Theory and Practice, Vol 1. Denver, CO: Bipedmed, LLC; 2013, p 27.

24. Coughlin MJ, Shurnas PS. Hallux rigidus. Grading and long-term results of operative treatment. J Bone Joint Surg Am 2003;85-A: 2072-2088.

25. Amis J, Jennings L, Graham D, Graham CE. Painful heel syndrome: Radiographic and treatment assessment. Foot Ankle 1988;9:91-95.

26. Kibler WB, Goldberg C, Chandler TJ. Functional biomechanical deficits in running athletes with plantar fasciitis. Am J Sports Med 1991; 19:66-71.

27. Riddle DL, Pulisic M, Pidcoe P, Johnson RE. Risk factors for Plantar fasciitis: A matched case-control study. J Bone Joint Surg Am 2003; 85-A:872-877.

28. Sweeting D, Parish B, Hooper L, Chester R. The effectiveness of manual stretching in the treatment of plantar heel pain: A systematic review. J Foot Ankle Res 2011;4:19.

29. Cole C, Seto C, Gazewood J. Plantar fasciitis: Evidence-based review of diagnosis and therapy. Am Fam Physician 2005;72:2237-2242.

30. Landorf KB, Menz HB. Plantar heel pain and fasciitis. BMJ Clin Evid 2008 Feb 5;2008. pii: 1111.

31. Sarrafian SK. Functional characteristics of the foot and plantar aponeurosis under tibiotalar loading. Foot Ankle 1987;8:4-18.

32. Wright DG, Rennels DC. A study of the elastic properties of plantar fascia. J Bone Joint Surg Am 1964;46:482-492.

33. Bolivar YA, Munuera PV, Padillo JP. Relationship between tightness of the posterior muscles of the lower limb and plantar fasciitis. Foot Ankle Int 2013;34:42-48.

34. Aranda Y, Munuera PV. Plantar fasciitis and its relationship with hallux limitus. J Am Podiatr Med Assoc 2014;104:263-268.

35. Wrobel JS, Armstrong DG. Reliability and validity of current physical examination techniques of the foot and ankle. J Am Podiatr Med Assoc 2008;98:197-206.

\section{Disclosure}

J.S.W. University of Michigan Medical School, Domino's Farm, Lobby G, Suite 1500, 24 Frank Lloyd Wright Dr., Ann Arbor, MI 48105. Address correspondence to: J.S.W.; e-mail: jswrobel@med.umich.edu

Disclosures related to this publication: grant, American Podiatric Medical Association (APMA), NIH; Disclosures outside this publication: payment for lectures, including service on speakers bureaus, lecture APMA meeting; travel/ accommodations/meeting expenses unrelated to activities listed, lecture APMA meeting

A.E.F. Dr. William M. Scholl College of Podiatric Medicine, Rosalind Franklin University of Medicine and Science, North Chicago, IL

Disclosures related to this publication: grant, American Podiatric Medical Association; nonfinancial support, Vasyli LLC provided prefabricated orthotics for use in this study at no cost
J.M.-B. Dr. William M. Scholl College of Podiatric Medicine, Rosalind Franklin University of Medicine and Science, North Chicago, IL Disclosures related to this publication: grant, American Podiatric Medical Association, NIH; nonfinancial support, Vasyli LLC provided prefabricated orthotics for use in this study at no cost

\section{J.F. Advocate Illinois Masonic Medical Center, Chicago, IL Disclosure: nothing to disclose}

R.T.C. Dr. William M. Scholl College of Podiatric Medicine, Rosalind Franklin University of Medicine and Science, North Chicago, IL

Disclosures related to this publication: grant, American Podiatric Medical Association; nonfinancial support, Vasyli LLC provided prefabricated orthotics for use in this study at no cost 
N.B. University of Michigan Medical School, Domino's Farm, Ann Arbor, MI Disclosure related to this publication: grant, American Podiatric Medical Association; nonfinancial support, Vasyli LLC provided prefabricated orthotics for use in this study at no cost

B.J. Dr. William M. Scholl College of Podiatric Medicine, Rosalind Franklin University of Medicine and Science, North Chicago, IL

Disclosures related to this publication: grant, American Podiatric Medical Association; nonfinancial support, Vasyli LLC provided prefabricated orthotics for use in this study at no cost
This study received funding from the American Podiatric Medical Association (APMA). It also received support from the National Institute of Diabetes and Digestive and Kidney Diseases (NIDDK), grant \#1T35DK074390. NIDDK and APMA had no role in the study design, data collection and analysis, decision to publish, or preparation of the manuscript.

Submitted for publication January 20, 2015; accepted September 3, 2015. 